\title{
VLBI OBSERVATIONS OF COMPACT EXTRAGALACTIC RADIO SOURCES WITH STEEP SPECTRA
}

\author{
V. K. Kulkarni \\ Radio Astronomy Centre (TIFR) \\ P. 0. Box 8, Doty 643001 , India \\ J. D. Romney \\ National Radio Astronomy Observatory \\ Edgemont Road, Charlottesville VA 22903-2475, U. S. A.
}

In recent years it has become clear that the common practice of dividing radio sources into two classes, compact flat-spectrum sources and extended steep-spectrum sources is too simplistic and that compact steep-spectrum sources (CSS) sources which seem to form a separate class in themselves, need to be taken into consideration. A majority of flat-spectrum sources are known to show asymmetric or D2 structures. In contrast to this many CSS sources when observed with subarcsecond resolution show symmetric structures typical of extended steep-spectrum sources. However, further high resolution observations of CSS objects are clearly needed to establish the structural differences, if any, between the two types of compact objects and to understand the interrelationship between the three classes of objects. In this connection we have undertaken a program to observe a sample of $18 \mathrm{CSS}$ sources using the European VLBI network. The sample was selected from the compilation by Peacock and Wall (1982), according to the following criteria: (1) flux density $>1.8 \mathrm{Jy}$; (2) largest angular size < 1 arc sec; and (3) steep high frequency spectral index $(>0.5)$ and high degree of low frequency spectral curvature. Our aim was to find out the structures common to these sources and their relation to more commonly observed "centimetre excess" compact objects. Here we will present the preliminary results.

We observed each source in our sample using the European VLBI Network (Onsala, Effelsberg, Dwingeloo/Westerbork, Jodrell) at 18/21 $\mathrm{cm}$, with a resolution of about $30 \mathrm{milliarcsec}$. The data were reduced at the Max Planck Institute for Radio Astronomy in Bonn. Based on consideration of aperture coverage and signal-to-noise ratio either a map was made or a model fitted to derive structural information about these sources. The maps are presented in Fig. 1.

The observations of our sample are not yet complete. Also we have data at only one frequency. Still based on our preliminary analysis we can draw some general conclusions. Our studies show that a large variety of structures is present. On the basis of our data 


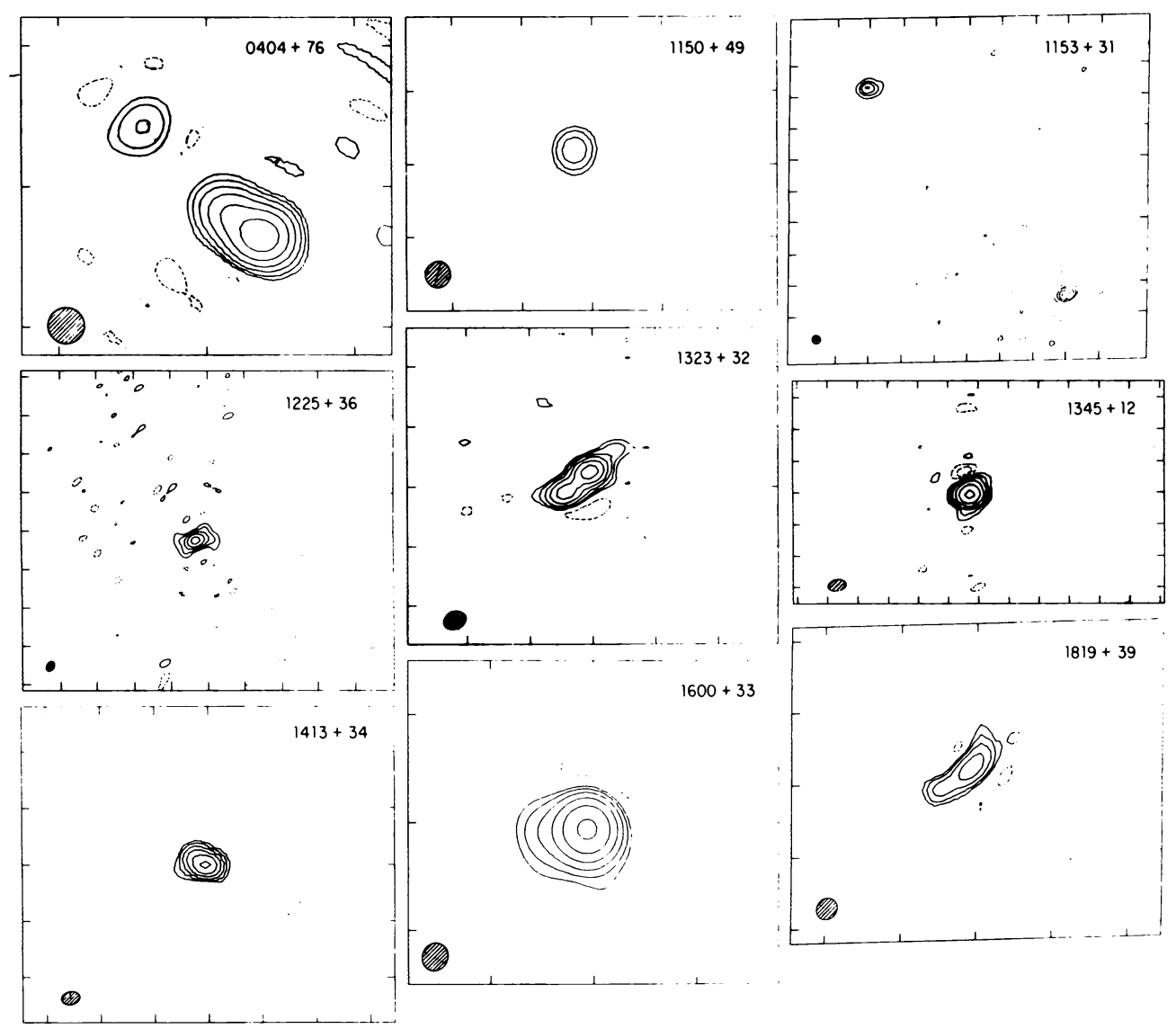

Fig. 1. VLBI maps of steep-spectrum compact sources. Tick marks are at intervals of 100 milliarcsec. Contours, in units of three times the r.m.s. noise in the residual map, are at levels of $-2,-1,1,2,4,8,16,32$, and 64 .

we attempted a rough morphological classification of 14 objects (for which observations are complete) in the present sample. Five sources were found to be double. Six sources were found to consist of single components, in some cases with extensions. Complex structures were found in three sources. Thus compact steep-spectrum sources show a wide range in structures.

\section{REFERENCES}

Peacock, J. A., and Wall, J. V., 1982, MNRAS, 198, 843. 